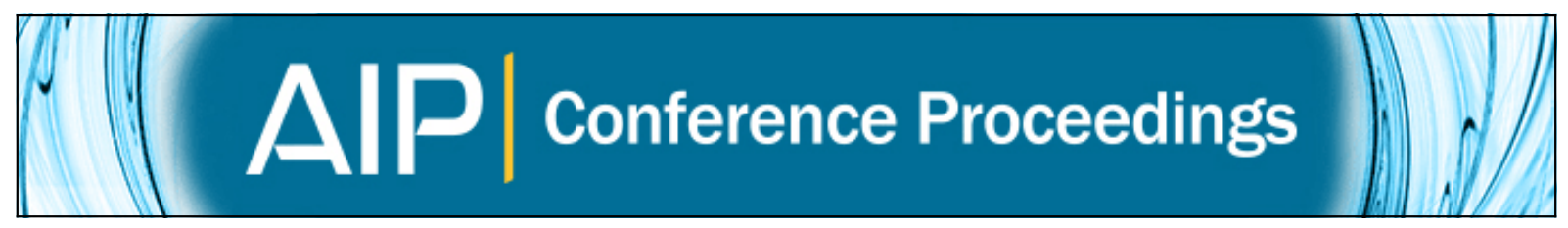

\title{
Fluid flow measurements using a scanning laser Doppler vibrometer
}

S. Vanlanduit, F. Prezniak, R. Longo, C. Vuye, and P. Guillaume

Citation: AIP Conference Proceedings 1253, 123 (2010); doi: 10.1063/1.3455449

View online: http://dx.doi.org/10.1063/1.3455449

View Table of Contents: http://scitation.aip.org/content/aip/proceeding/aipcp/1253?ver=pdfcov

Published by the AIP Publishing

\section{Articles you may be interested in}

Surface wave measurements using a single continuously scanning laser Doppler vibrometer: Application to elastography

J. Acoust. Soc. Am. 133, 1245 (2013); 10.1121/1.4789929

Broadband measurement of translational and angular vibrations using a single continuously scanning laser Doppler vibrometer

J. Acoust. Soc. Am. 132, 1384 (2012); 10.1121/1.4740473

Using laser Doppler vibrometry to measure capillary surface waves on fluid-fluid interfaces

Biomicrofluidics 4, 026501 (2010); 10.1063/1.3353329

Absorption measurement of acoustic materials using a scanning laser Doppler vibrometer J. Acoust. Soc. Am. 117, 1168 (2005); 10.1121/1.1859233

Nonperturbing measurements of spatially distributed underwater acoustic fields using a scanning laser Doppler vibrometer

J. Acoust. Soc. Am. 115, 187 (2004); 10.1121/1.1635841 


\title{
Fluid flow measurements using a scanning laser Doppler vibrometer
}

\author{
S. Vanlanduit, F. Prezniak, R. Longo, C. Vuye and P. Guillaume \\ Deptartment of Mechanical Engineering, \\ Vrije Universiteit Brussel, Pleinlaan 2, B-1050, Brussels, Belgium \\ e-mail: steve.vanlanduit@vub.ac.be
}

\begin{abstract}
The laser Doppler vibrometer (LDV) is an instrument based on a heterodyne interferometer that can be used to measure vibrations at a location on a structure. By moving the laser beam using scanning mirrors a full field vibration measurement can be performed. While the main purpose of the scanning LDV is to measure vibrations, recently several authors proposed to use the LDV for flow and sound measurements. The idea is to aim the laser vibrometer at a rigid block. When a pressure field is present between the LDV head and the rigid block this will result in changes of the refraction index that are related to the measured Doppler shift. Because no seeding particles are needed, the method opens new opportunities to measure phenomena that were previously difficult to measure. Unfortunately, the current LDV measurement techniques are only able to measure dynamic flow fields (both turbulent and coherent).

In this paper we present a novel method that is able to measure a steady-state velocity flow field. The technique is based on the use of an ultrasound field that is generated in addition to the flow field. At locations where a non-zero flow velocity is present, the ultrasonic wave fronts will be phase shifted. The ultrasound fields can be measured using a scanning LDV. By calculating the phase shift from the measurements the flow velocity can be obtained. The proposed method will be validated on measurements of a jet
\end{abstract}

Keywords: laser Doppler vibrometer, fluid flow measurement.

PACS: $47.80 . \mathrm{Cb}, 47.80 . \mathrm{Jk}$

\section{INTRODUCTION}

Optical measurement techniques are often used for fluid flow measurements. The laser Doppler anemometer (LDA) is an instrument that is able to perform time resolved measurements of the velocity at a single location [1]. Two crossing laser beams are used to generate a measurement volume with fringes. When a particle passes through the measurement volume, a Doppler burst signal is generated. The frequency of the burst can be related to the velocity of the particle and hence of the flow at the position of the measurement volume. The LDA measurement technique is attractive because it enables measurements in a large frequency band (from DC to several $\mathrm{kHz}$ ). However, when full field measurement of the flow field is necessary, the LDA technique is too time consuming. In that case the Particle Image Velocimetry (PIV) method [2] is a more attractive alternative. In PIV a laser sheet is generated using a cylindrical lens. Tracer particles are used to seed the flow. Images of the seeded flow are captured at two time instances. Then a correlation technique is used to calculate the displacement of the tracer particles in between the two recorded frames. With the introduction of high speed camera's it became possible to perform full field flow measurements at frequencies up to several $\mathrm{kHz}$.

Though both LDA and PIV became standard measurement techniques in experimental fluid mechanics their use has an important drawback. The flow should be properly seeded in order to perform reliable measurements [3]. This can be a problem in case of large measurement volumes (wind tunnel testing), high velocities, high temperatures, etc. In this paper we present a novel technique that does not require seeding particles. The method is based on the use of a laser Doppler vibrometer to measure the density variations of a flow field. 


\section{THE PROPOSED MEASUREMENT TECHNIQUE}

The laser Doppler vibrometer (LDV) is an instrument based on a heterodyne interferometer that was developed in the seventies to measure vibrations [4]. Because of its contactless nature it can be used in hostile environments. Nowadays LDVs are available to measure in a very broad frequency band (from almost DC up to $30 \mathrm{MHz}$ ). The instrument is applied on very small microstructures but also on large structures like bridges.

Since the early nineties several researchers also used the LDV to measure acoustic fields $[5,6,7]$. Instead of aiming the LDV beam onto a measurement point on a vibrating structure the beam is directed to a rigid reflector. It can be shown that the Doppler signal is given by:

$v(t)=\frac{d s(t)}{d t}=\frac{d(n(t) z)}{d t}$

with $S(t)$ the optical path length, $n(t)$ the refraction index of the medium and $z$ the laser source - object distance. The right-hand numerator can be split in two terms:

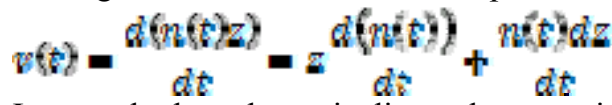

In case the laser beam is directed onto a rigid non-vibrating block the second term in this equation is zero. In case a pressure wave is present between the rigid block and the laser source, it can be shown that:

$\frac{d(n t))}{d t}=\frac{n_{q}-1 d p(t)}{d t}$

Therefore, the Doppler signal will be proportional to the time derivative of the pressure.

Since pressure derivatives are measured, the method described above can also be used to measure flow fields. In [8] it was applied to measure turbulent flow fields. Other papers propose LDV measurement results for coherent structures like vortices in the wake of bluff bodies $[9,10]$.

The main limitation of the use of the LDV to measure flow fields is that it can only be used for dynamic measurements. Indeed, the Doppler signal is proportional to the time derivative of the pressure signal. This means that the sensitivity increases with the frequency (if one assumes a constant pressure amplitude).

In this paper we propose an alternative method to measure a flow field using a scanning LDV. In contrast to the existing LDV flow measurement technique, the method can be used to measure steady state velocity fields. The principle is similar to the operating mechanism of a sonic anemometer [11]. A sonic anemometer contains two ultrasound transducers for each measurement direction (see e.g. the principle of a 2-dimensional sonic anemometer in Figure 1). An ultrasound pulse is generated by one of the transducers. The other transducer receives the signal and measures the time delay between the sent and received pulse. In case no wind is present, this time delay is related to the speed of sound $C$ of the medium. When a wind velocity $V$ is present, the delay is proportional to $V+\varepsilon$.

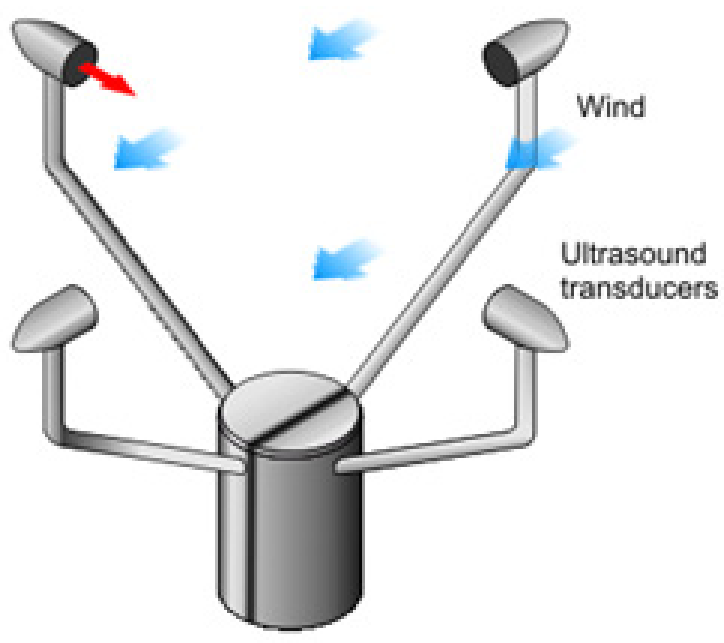

FIGURE 1. Measurement principle of the sonic anemometer. 
In what follows we will also create an ultrasound field in addition to the flow field that we want to measure. Instead of measuring the time delay at one location with a receiving ultrasound transducer in the case of a sonic anemometer, we will use the scanning LDV to measure the full field ultrasound field. From the phase shift of the ultrasound field we can calculate the velocity at each measurement location. The method will be illustrated in the next section on a round jet.

\section{VALIDATION EXPERIMENT}

\section{Measurement setup}

The proposed procedure will be illustrated on a round jet that is generated by connecting a $2 \mathrm{~cm}$ diameter tube to a supply hose that is connected to the laboratory pressurized air supply (see measurement setup in Figure 2). An ultrasound transducer at $40 \mathrm{~cm}$ below the jet outlet is used to create a cylindrical wave between two plexi-glass plates. A rigid aluminum plate is placed behind the jet outlet (the plate is covered with $3 \mathrm{M}$ retro-reflective tape).

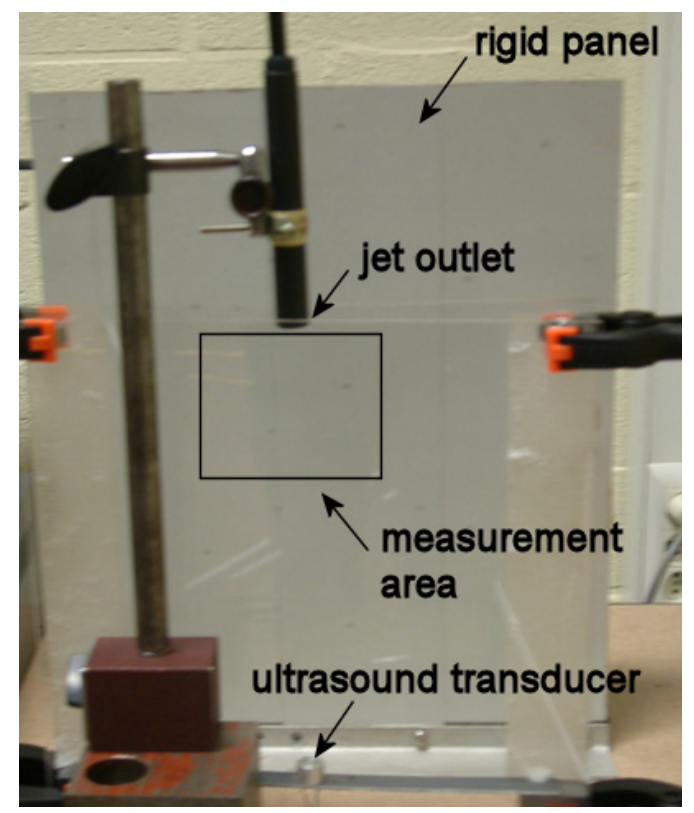

FIGURE 2. Measurement principle setup of the round jet scanning LDV measurement.

The measured ultrasound fields with and without the jet are shown in Figure 3. By calculating the relative phase shift between the ultrasound waves the velocity field can be reconstructed:

$$
\theta_{x}=\operatorname{angle}\left(\frac{v^{2}}{v^{2}}\right)
$$

with $v^{x}$ the measured ultrasound field with a jet inlet at air pressure $x$. Remark that the measured ultrasound fields are complex valued (since they are related to the generator signal of the ultrasound transducer). The phase shifts that are calculated are wrapped between - pi and + pi. They can be unwrapped by removing all steps larger than $\pi$. This can be done in the spatial domain or in the time domain if more than two measurements are available [12]. The results of the phase shifts before and after (temporal) unwrapping are shown in Figure 4. 

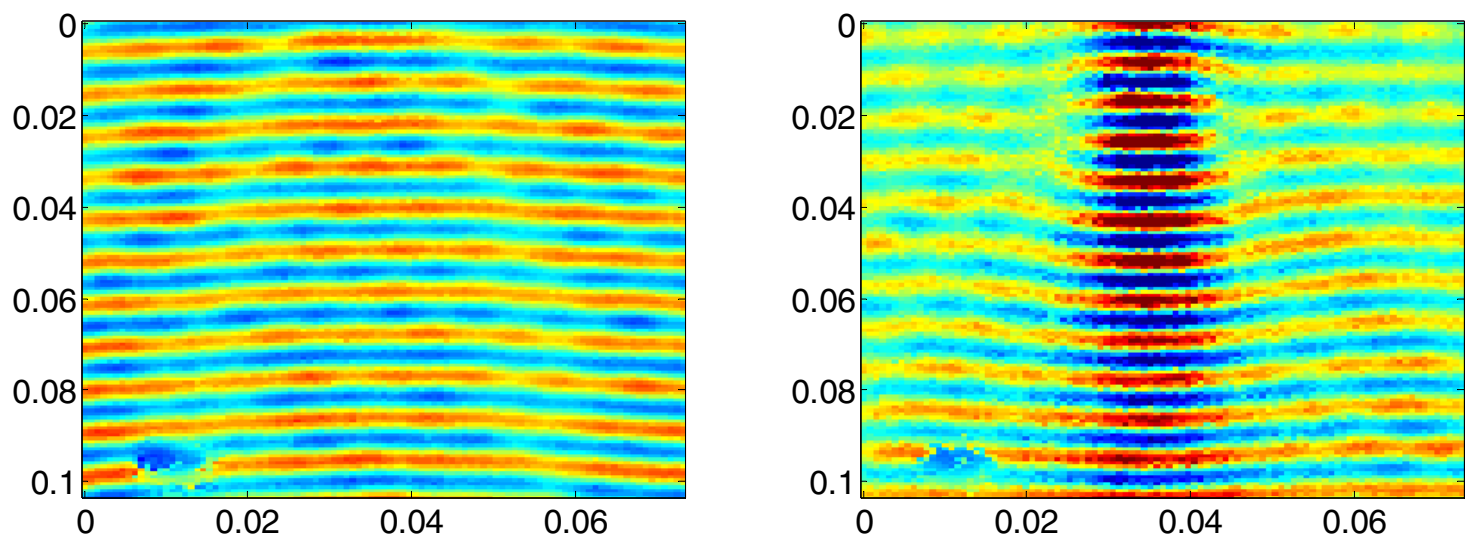

FIGURE 3. Measured ultrasound waves without jet (left) and with a 5 bar jet (right).
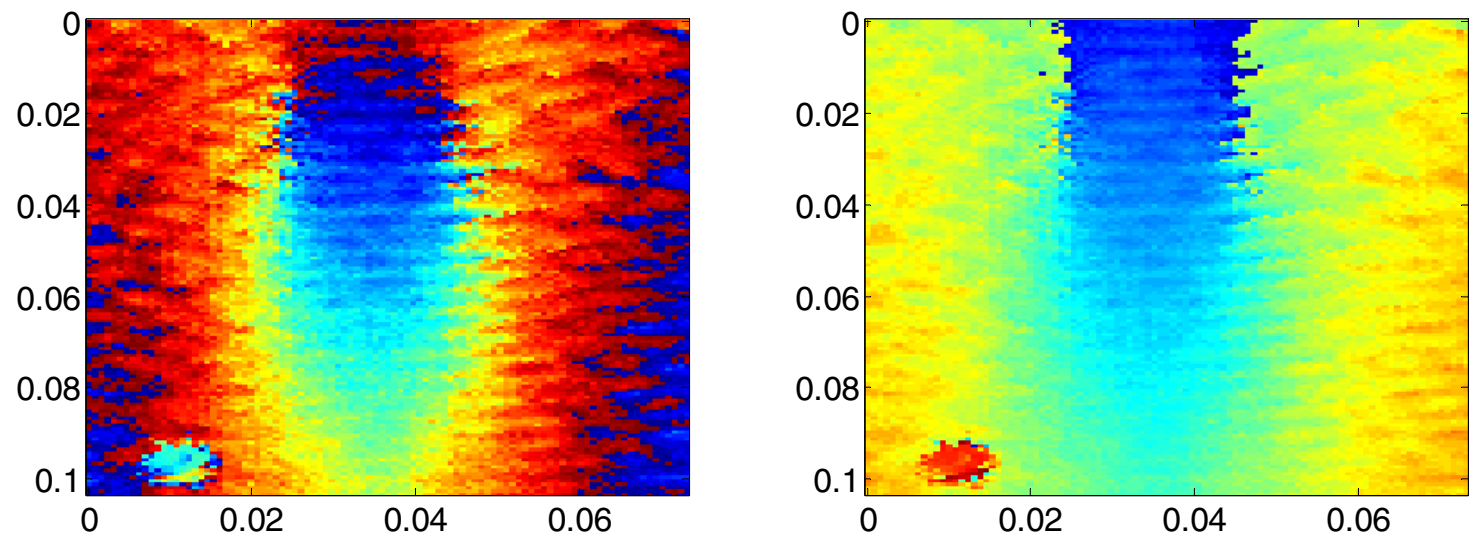

FIGURE 4. Left: phase shift between ultrasound field with and without jet (see Figure 3). Right: unwrapped phase angle.
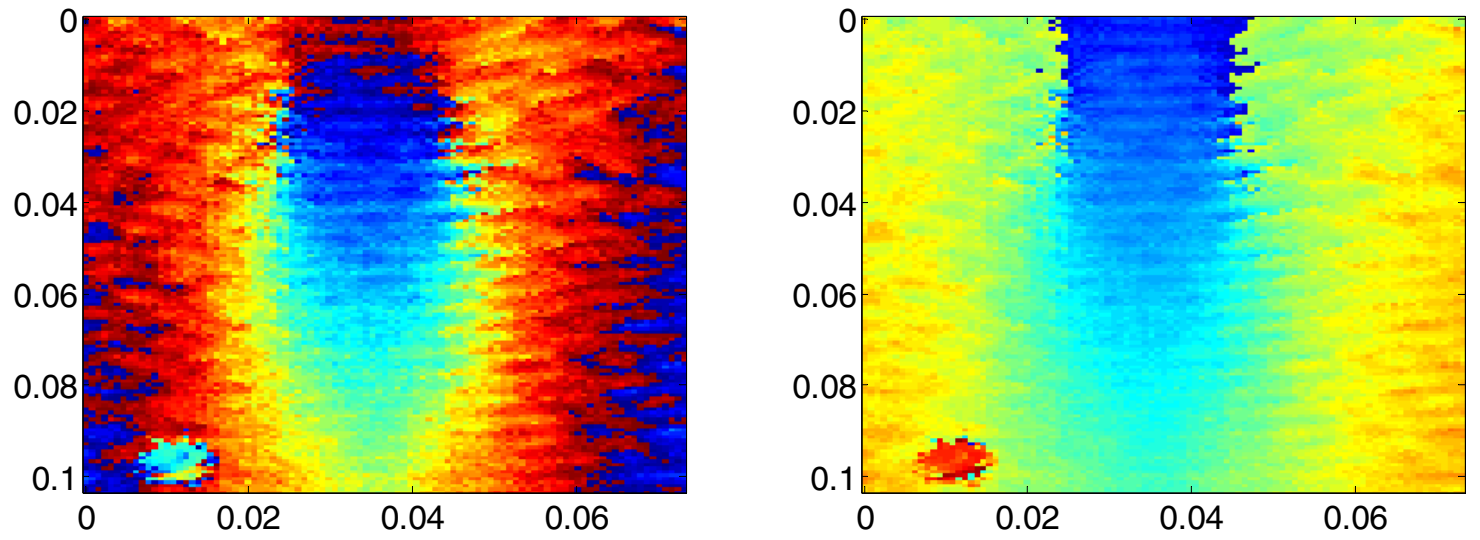

FIGURE 5. Left: phase shift between ultrasound field with and without jet (see Figure 3). Right: unwrapped phase angle. 


\section{Jet flow characteristics}

The round jet is a frequently studied flow in experimental fluid mechanics. PIV experiments of the averaged flow (as well as the turbulence) are reported in function of the energy of the jet $[13,14]$. In general two zones can be differentiated in the jet flow: the zone of establishment where the flow has a flat-top like shape and the established flow area where the averaged velocity is Gaussian (see Figure 6). When the velocity is plotted in function of the distance to the orifice (on a logarithmic scale) a 1/x law can be observed in the established zone (see Figure 6).
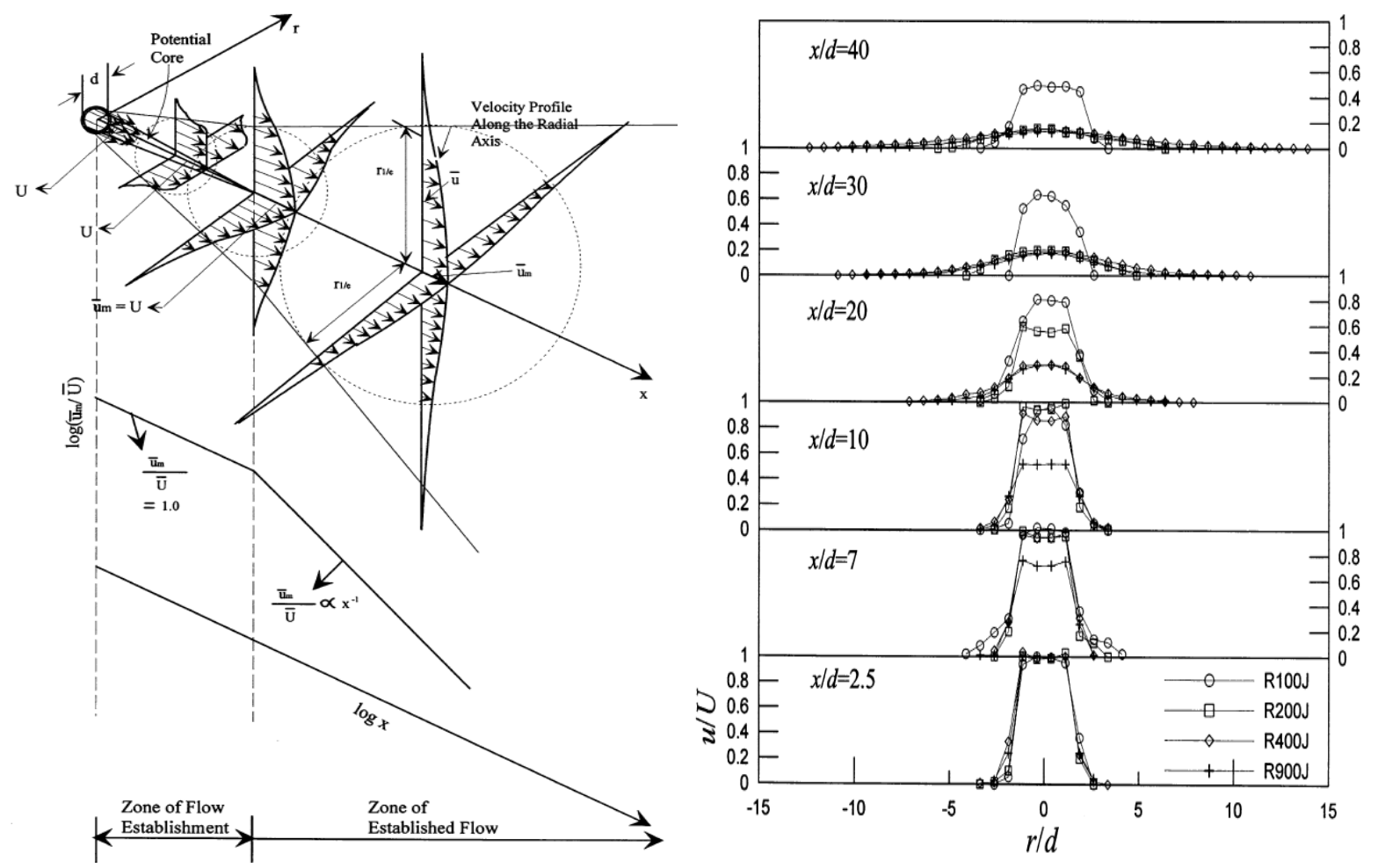

FIGURE 6. Shape of the average velocity field of a round jet, reprinted from [1313]. Left: schematic 3D view. Right: PIV measurement results.

\section{Measurement results}

The measurements of the round jet with increasing inlet pressure using the proposed method are shown in Figure 7. Slices at different distances from the jet outlet are given in Figures 8 and 9 . When comparing the measurement results in Figure 8 with the PIV measurements in Figure 6 it can be seen that the shape of the jet corresponds well: near the outlet the jet has a flat-top shape while further away a Gaussian flow field is present. 


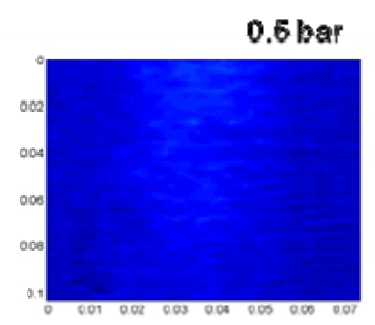

2.6 bar

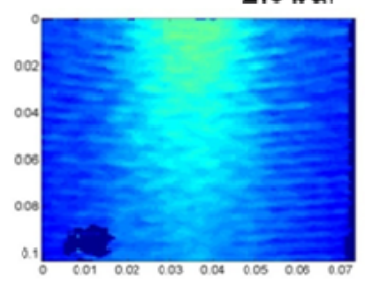

$4.5 \mathrm{bar}$

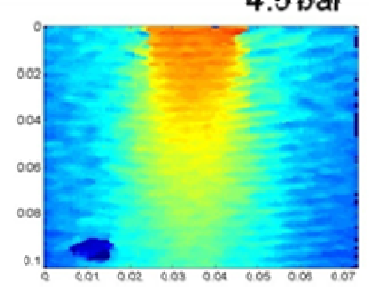

1 bar

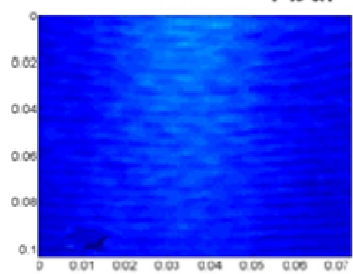

3 bar

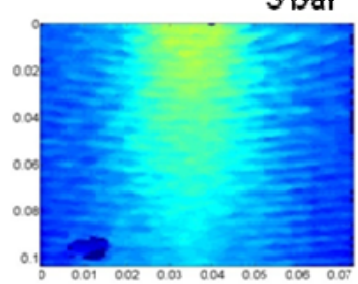

5 bar

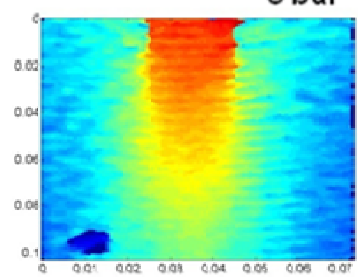

1.5 bar

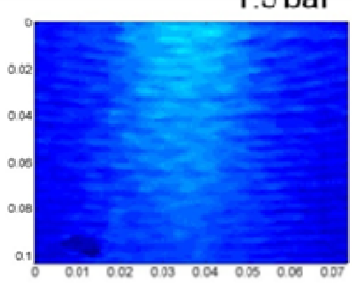

3.5 bar

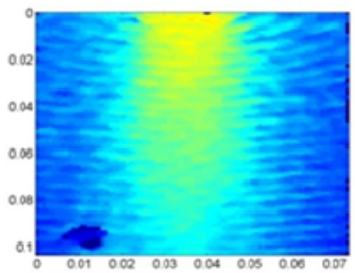

5.5 bar

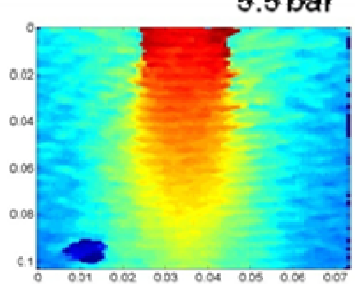

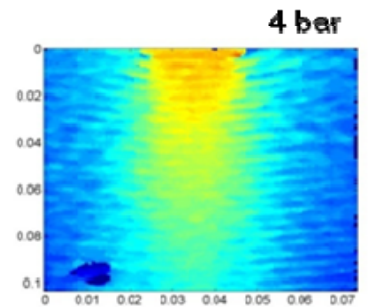

2 bar

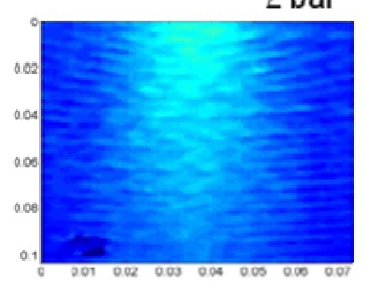

4 ber

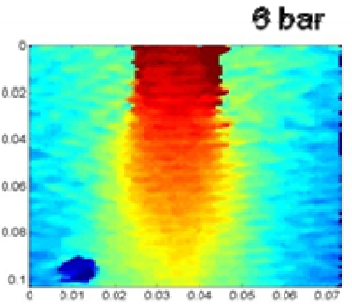

FIGURE 7. Velocity fields for increasing inlet pressure measured using the proposed method.

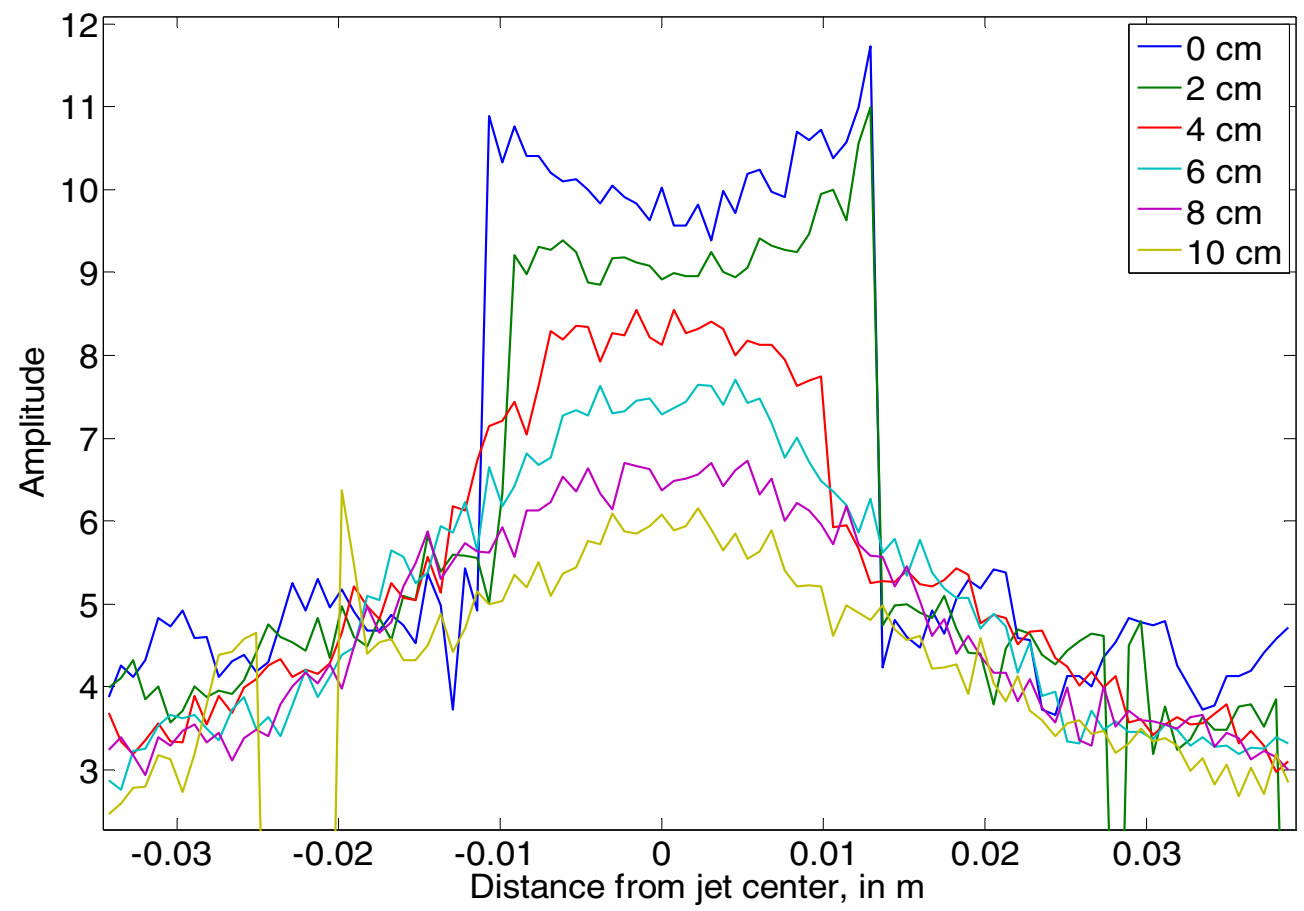

FIGURE 8. Horizontal slices of the measured flow field in Figure 7 (corresponding to the 6 bar image). 


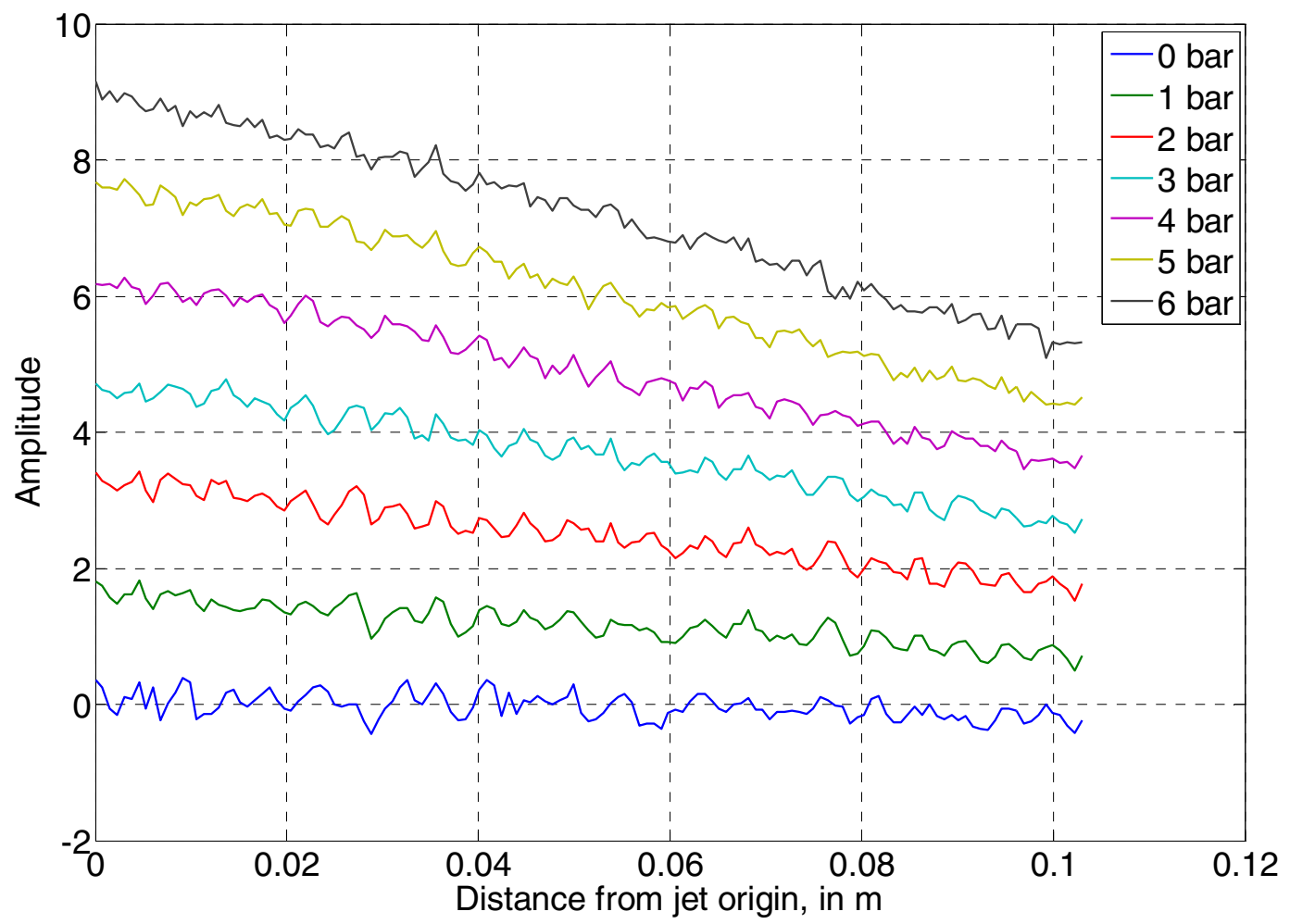

FIGURE 9. Vertical slices of the measured flow field in Figure 7 (corresponding to the 6 bar image).

\section{ACKNOWLEDGMENTS}

This research has been sponsored by the Flemish Institute for the Improvement of the Scientific and Technological Research in Industry (IWT), the Fund for Scientific Research - Flanders (FWO) Belgium. The authors also acknowledge the Flemish government (GOA-Optimech+) and the research council of the Vrije Universiteit Brussel (OZR) for their funding.

\section{REFERENCES}

1. Hussein HJ, Capp Sp, George Wk, Velocity-Measurements In A High-Reynolds-Number, Momentum-Conserving, Axisymmetrical, Turbulent Jet, Journal Of Fluid Mechanics, 258, 31-75, 1994.

2. Keane RD, Adrian RJ, Theory Of Cross-Correlation Analysis Of PIV Images, Applied Scientific Research, 49(3), 191-215, 1992.

3. Melling A, Tracer particles and seeding for particle image velocimetry, Measurement Science and Technology, 8(12), 14061416, 1997.

4. Halliwell NA, Laser-Doppler measurement of vibrating surfaces: A portable instrument, Journal of Sound and Vibration, 62(2), 22 January 1979, Pages 312-315.

5. Zipser L, Franke H, Visualisation and measurement of acoustic and fluidic phenomena using a laser-scanning vibrometer, 5th International Conference on Vibration Measurements by Laser Techniques, JUN 18-21, 2002 SPIE Proceedings, Volume: 4827, p. 192-198, 2002

6. Harland AR, Petzing JN, Tyrer JR, Nonperturbing measurements of spatially distributed underwater acoustic fields using a scanning laser Doppler vibrometer, Journal of the Acoustical Society of America, 115(1), 187-195, 2004.

7. Vanherzeele J, Vanlanduit S, Guillaume P, Acoustic source identification using a scanning laser Doppler vibrometer, Optics and Lasers in Engineering, 45(6), 742-749, 2007.

8. Mayrhofer N, Woisetschlager J, Frequency analysis of turbulent compressible flows by laser vibrometry, Experiments in fluids, 31(2), 153-161, 2001. 
9. Woisetschlager J, Mayrhofer N, Hampel B, et al., Laser-optical investigation of turbine wake flow, Experiments in Fluids, 34(3), 371-378, 2003.

10. Vanherzeele, J, Brouns, M, Castellini, P, Guillaume, P, Martarelli, M, Ragni, D, Tomasini, EP, Vanlanduit, S, Flow characterization using a laser Doppler vibrometer, Optics and Lasers in Engineering 45(1), 19-26, 2007.

11. Anfossi D, Oettl D, Degrazia G, et al, An analysis of sonic anemometer observations in low wind speed conditions, Boundary-layer Meteorology, 114(1), 179-203, 2005.

12. Huntley, Jm; Saldner, H, Temporal Phase-Unwrapping Algorithm For Automated Interferogram Analysis, Applied Optics, 32(17), 3047-3052 Published: Jun 101993.

13. Kwon SJ, Seo IW, Reynolds number effects on the behavior of a non-buoyant round jet, Experiments in Fluids, 38: 801$812,2005$.

14. Bi, WT; Sugii, Y; Okamoto, K, et al., Time-resolved proper orthogonal decomposition of the near-field flow of a round jet measured by dynamic particle image velocimetry, Measurement Science \& Technology, 14(8), L1-L5, 2003. 